\title{
Gross Values: Investigating the role of disgust in bioethics
}

Researchers have interpreted effects of disgust on condemnation of "purity" violations as evidence that emotions amplify or even constitute moral judgments. However, subsequent replication failures raise uncertainty about the link between disgust and judgment severity. How disgust affects attitudes toward biotechnologies that threaten purity remain particularly underexplored. In the present study, participants $(N=600)$ viewed disgusting, frightening, or neutral pictures, rated the moral acceptability of biomedical technologies, and completed questionnaire measures of trait disgust. We find a small negative effect of induced disgust (but not fear) on the acceptability of 'existing' biotechnology, but not 'future', 'agricultural', or 'termination' biotechnologies. The one significant effect was too small to change pre-existing opinions and would not have survived a correction for multiple tests. Although trait disgust had mostly negative relationships with moral acceptability of biotechnologies, it did not moderate the effect of observing disgusting photos on biotechnology judgments. The larger, more consistent effects for trait disgust suggest that either (a) measures of trait disgust and moral attitudes share a source of method variance or (b) incidental, visual manipulations are too weak to capture the true effect of disgust on moral judgments.

Keywords: disgust; morality; moral emotions; bioethics 


\section{Introduction}

Imagine you are waiting for your results at a doctor's office. Your eyes wander over posters depicting smoke-damaged lungs, lurid skin diseases, and dissected brains. You feel queasy. Then the doctor returns with your results - they are not good - and recommends a novel invasive procedure. You are repulsed by the idea, it seems unnatural, disgusting... wrong. You are about to reject the idea... but should you trust your gut reaction?

Recent research in moral psychology has argued that the emotion of disgust plays a substantial role in moral judgement. According to this theory, disgust evolved in humans to motivate avoidance of pathogens, parasites, and other disease-vectors (Schaller \& Park, 2011). This psychological system also motivates avoidance of social hazards - 'unclean' people and practices. It has been argued this has given rise to a distinct domain of morality concerned with defending and maintaining bodily and spiritual 'purity' (Haidt \& Joseph, 2004). This theory predicts that inductions of disgust and variation in 'trait' disgust should influence moral judgments.

\section{Does disgust influence moral judgment?}

A series of early experiments suggested that experimentally inducing disgust or feelings of cleanliness increases and decreases moral condemnation, respectively (Schnall, Benton, \& Harvey, 2008; Wheatley \& Haidt, 2005). Later experiments report that disgust only affects judgments of 'purity' violations such as counter-normative sexual behavior (Horberg, Oveis, Keltner, \& Cohen, 2009; Olatunji, Puncochar, \& Cox, 2016). Similarly, correlational studies have shown that trait disgust primarily predicts condemnation of purity violations (Inbar, Pizarro, \& Bloom, 2009; Wagemans, Brandt, and Zeelenberg, 2018).

However, a meta-analysis found that the effect of experimentally induced disgust on moral judgements is small $(d=0.11)$ and, after accounting for publication bias, possibly nil $(d=-0.01 ;$ Landy \& Goodwin, 2015). Since then, large replications of key studies in the 
meta-analysis have also failed to replicate, even among groups thought to be especially sensitive to disgust (Ghelfi et al., 2018; Johnson et al., 2016). Recent positive effects of induced disgust have been limited to particular types of moral scenarios (Tracy, Steckler, \& Heltzel, 2019; van Dijke, van Houwelingen, De Cremer, \& De Schutter, 2018; Wisneski \& Skitka, 2017). The mixed picture painted by experiments suggests that the more robust association between trait disgust and moral judgments is partly spurious. Indeed, one recent study suggests that associations between trait disgust and negative moral judgments merely reflects generalized negativity (Landy \& Piazza, 2019).

\section{How does disgust affect moral judgments?}

Even if disgust does affect moral judgments, it need not follow that emotions like disgust are necessary or sufficient to generate moral condemnation, as some commentators have theorized (Nichols, 2002; Prinz, 2007). Even landmark incidental disgust experiments did not produce strong enough effects to change participants' opinions from the "approval" or "neutral" side of rating scales to the "disapproval" side, implying that disgust at most amplified preexisting attitudes (May, 2014). Furthermore, such amplification could be mediated by non-emotional processes, in which case disgust would be best characterized as one factor among indefinitely many that can affect the antecedents of moral judgments. For example, disgust inductions may change people's understanding of the act they are evaluating, perhaps by drawing their attention to its potential harm (Huebner, Dwyer, \& Hauser, 2009). Alternatively, participants may resent being intentionally exposed to noxious stimuli, and express their bad mood by making more negative moral judgments (Landy \& Goodwin, 2015). On these views, asking people to pay attention to a certain aspect of an act while evaluating it or insulting participants before having them judge scenarios would bring about the same effects as disgust inductions via the same psychological processes. Thus, significant effects of disgust inductions on moral judgments are not evidence that disgust has 
an integral role in moral judgments unless one can rule out that participant's mood, and understanding of the acts they are judging, do not account for condition differences. One step towards ruling out these confounds is establishing measurement invariance across disgust and control conditions. Measurement invariance would hold if disgust inductions reduced reports of moral acceptability by changing people's attitudes towards moral biotechnologies rather than merely changing the relationships between people's attitudes and how they report those attitudes (Gregorich, 2006).

\section{The Present Study}

The present study investigates the roles of induced and trait disgust on judgments of controversial biological and medical technologies — the province of bioethics. Bioethical issues provide a good test case for the effects of disgust because most of them involve a purity violation: Many worry that gene editing compromises the "essence" of persons, pesticides contaminate the environment, genetically modified organisms (GMOs) do not possess the nutrition available from "natural" foods, and so on (Clifford \& Wendell, 2016; De Witt, Osseweijer, \& Pierce, 2017). Whether disgust influences the perceived acceptability of biotechnologies also has important practical implications. Disgust may influence healthcare provision, which is set in locations (e.g. hospitals, clinics) in which disgust elicitors abound. And disgust may stall debates about whether to legalize or subsidize biotechnologies insofar as emotional reactions are immune to cost-benefit arguments.

Research on the role of disgust in biotechnology opposition has yet to reach definitive conclusions. One study found that state and trait level disgust predict "absolutist" (that is, insensitive to risks and benefits) opposition to GMOs (Scott, Inbar, \& Rozin, 2016). Followup studies show that participants use the word "disgust" metaphorically to express a wariness of unforeseen negative consequences of novel biotechnologies (Royzman, Cusimano, \& Leeman, 2017). Clifford and Wendell (2016) found significant associations between trait 
disgust and a handful of biotechnologies. However, main effects of induced disgust were non-significant across the board. There was inconsistent evidence that induced disgust caused more condemnation of GMOs among those more sensitive to pathogen disgust.

The current experiment has a similar protocol to Study 3 of Clifford and Wendell (2016) but introduces several improvements. First, Clifford and Wendell (2016) compared their disgust induction only to an affect-neutral control. We followed Horberg et al. (2009) in also including a fear condition to test whether disgust affects moralization simply by inducing negative affect (thereby having a similar effect to fear) or through some unique property (thereby having a different effect than fear). Second, Clifford and Wendell (2016) treated each biotechnology as a separate dependent variable and examined the effects of trait disgust, induced disgust, and their interaction on each outcome. In spite of the large number of tests, the authors made no attempt to control the family-wise error rate. We planned a limited number of tests and report the family-wise error rate. The unplanned sensitivity tests we conducted were also designed to minimize false positives. Third, we recruited a sample that was larger $(N=600)$ than their two experiments combined (Study $1 N=210$, Study $3 N=$ 311; Study 2 did not include an experimental manipulation). Fourth, Clifford and Wendell (2016) did not correct for measurement error. In contrast, we modeled biotechnologies as latent variables, which have perfect reliability.

Our latent variable approach also enabled us to test for measurement invariance across experimental conditions. This approach addresses concerns that skeptical commentators often raise when interpreting positive results. In particular, tests of measurement invariance allow for an explicit test of whether disgust inductions reduce judgments of moral acceptability by changing the measurement process rather than directly changing people's attitudes towards moral biotechnologies (Gregorich, 2006). If the biotechnology items possess configural invariance, then we can infer that the same constructs 
underlying judgments of biotechnologies are being measured across experimental conditions. We test this assumption using multi-group confirmatory factor analysis, which fits the same factor structure in each condition, but allows factor loadings and items to be freely estimated within each condition. Configural invariance is achieved if the multi-group model has good model fit. If the moral judgment items possess metric invariance, then participants in different conditions likely possess the same understanding of the acts that they are judging. Thus, condition differences in moral judgments would not be attributable to disgust simply focusing participants' attention on features other than those to which they would normally attend. We test metric invariance by constraining the factor loadings equal across conditions in a multi-group model that possesses configural invariance. We infer metric invariance when equalizing factor loadings does not degrade model fit relative to the configural model. If the items also possess scalar invariance, then condition-mean differences reflect genuine changes in the moral judgment process rather than mere changes in how participants respond to questionnaire items. We test scalar invariance by observing whether model fit declines after constraining item intercepts equal across conditions in the metric invariant model.

\section{Methods}

\section{Preregistration}

Our planned sample size, protocol, and analysis plan were preregistered and are available here: https://osf.io/j36ex. Some details of our analyses were not pre-registered but were consistent with conventional applications of structural equation modeling, such as using cut-offs of approximate fit indices (RMSEA $\leq .06, \mathrm{TLI} \geq .95$, and $\mathrm{SRMR} \leq .08$ are adequate; RMSEA $\leq .10, \mathrm{TLI} \geq .90$, and SRMR $\leq .10$ are mediocre) to evaluate model fit adequacy (Hu \& Bentler, 1999). However, some methodologists find conventional practices too lax. In the "Sensitivity Analysis" section of the supplemental analyses (https://osf.io/d49mk/) we present results from unplanned analyses in which we (a) aggregate biotechnologies without 
making assumptions about their latent structure, (b) treat each biotechnology as an independent outcome, and (c) test hypotheses only after addressing model misfit. Because none of the sensitivity analyses generated significant results that were not also found in the primary analyses, we do not report on them further here.

\section{Recruitment}

600 participants $\left(459\right.$ women; $\left.M_{\mathrm{age}}=48.84, S D_{\mathrm{age}}=17.31\right)$ based in the United States, were recruited via Qualtrics, in exchange for $£ 4.25$, to complete a questionnaire. Our sample size gave us $80 \%$ power to detect condition effects as small as $d=.24$ and correlations as small as $r=.11$, assuming two-tailed tests with an alpha of .05. We calculated statistical power using G*Power (Faul, Erdfelder, Buchner, \& Lang, 2009). Sample size was determined before any data analysis.

\section{Procedure}

No other measures or manipulations beyond those described below were administered. First, participants observed in sequence 20 pictures sourced from the International Affective Picture System (IAPS), a well-established picture set of emotional stimuli for exclusively scholarly interests (Libkuman, Otani, Kern, Viger, \& Novak, 2007). There were three conditions. Participants saw either twenty examples of disgusting pictures (e.g., vomit and wounds), frightening pictures (e.g., guns and snakes), or neutral pictures (e.g., cups and furniture).1 The items within this, and each subsequent, section of the procedure were presented in random order.

After observing each picture, participants rated their reaction on a 7-point rating scale, (1 = "Extremely unpleasant", 2 = "Very Unpleasant", 3 = "Unpleasant", 4 = "Neutral", 5 =

1 As noted by Landy and Goodwin (2015), using the IAPS rather than stimuli involving people performing disgusting acts allows us to circumvent the possibility that disgust increases condemnation of specific people who engage in the act under consideration rather than of the act per se. 
"Pleasant", 6 = "Very Pleasant", 7 = "Extremely pleasant"). These ratings were averaged and served to verify whether the disgust and fear conditions elicited more negative affect than the neutral conditions. Although ratings of pleasantness cannot verify that the disgust pictures primarily elicited disgust and the fear pictures primarily elicited fear, IAPS photos have been validated for eliciting specific emotions, including disgust and fear (Mikels, Fredrickson, Larkin, Lindberg, Maglio, \& Reuter-Lorenz, 2005). Moreover, checking that the intended emotional response occurred can make transparent the hypotheses under study to the participants (Ejelöv \& Luke, 2020). In contrast, having participants report on the pleasantness of the photos does not confound the unique effects of the disgust or fear condition.

Second, participants rated the moral acceptability of twenty biomedical technologies on a 5 -point rating scale $(1=$ Completely unacceptable; $2=$ Unacceptable; $3=$ Neither acceptable nor unacceptable; 4 = Acceptable; 5 = Completely acceptable). (See Table 1 for descriptive statistics grouped by condition.) We defined "moral acceptability" for participants as "something that, from your moral viewpoint, a person can do without being wrong." Items were collated from a review of previous studies of bioethics (Graham et al., 2011; Haidt, 2007; Horberg et al., 2009; Schnall, Benton, \& Harvey, 2008; Pew Research Center, 2015; National Science Foundation, 2012). See the supplementary materials (https://osf.io/n4az9/files/) for exact wording of the instructions and items.

Third, participants completed the "Pathogen Disgust" (omega $=.85 ; M=4.17, S D=$ 1.22 ) and "Sexual Disgust" (omega $=.86 ; M=3.58, S D=1.53$ ) sub-scales of the Three Domain Disgust Scale (TDDS), a widely-used questionnaire measure of trait disgust (Tybur, Lieberman, \& Griskevicius, 2009). Participants were asked to rate how disgusting they found the concepts described in the questionnaire from "Not at all disgusting" to "Extremely disgusting” on a 7-point rating scale. 
Fourth, participants completed the Judgment scale of the Morality as Cooperation Questionnaire (MAC-Q; Curry, Jones Chesters, \& Van Lissa, 2019), as well as demographic questions about political identity, age, gender, religious affiliation, marital status, nationality, number of children and grandchildren, and education. These variables were not analysed in the present study.

Finally, participants were invited to provide open-ended feedback about the survey. We coded feedback for pejorative comments about the IAPS photos $(n=11$ out of 190 participants in the disgust condition) to empirically examine whether anger at exposure to noxious stimuli could explain significant effects of the disgust condition (Landy \& Goodwin, 2015). There were no complaints about the fear photos or neutral photos.

\section{Results}

All tests of hypotheses were conducted in R version 3.5.1 (R Core Team, 2019) using the lavaan (Rosseel, 2012) and psych (Revelle, 2018) packages. Full Information Maximum Likelihood was used to handle missing data, and robust standard errors were used to handle violations of multivariate normality. There were no data exclusions. We treated rating scale items as continuous because they all had at least five categories (Rhemtulla, Brosseau-Liard, and Savalei, 2012). All tests were two-tailed with an alpha of .05. Data and syntax are available in the supplementary materials.

\section{Factor Structure}

We used exploratory factor analysis with an oblimin rotation to determine the factor structure of biotechnology judgments. We settled on four factors, and then affirmed measurement invariance across experimental conditions. For full statistical details see the supplemental analyses (https://osf.io/d49mk/).

We labeled the first factor Existing; it comprises biotechnologies that are already available: acupuncture, alternative medicine, artificial organs, vaccines, eating meat, stem 
cell research, using genetically modified plants to create a replacement for gasoline, using robots to assist with surgery, and treating very sick patients with experimental drugs (with their consent).

We labeled the second factor Future; it comprises biotechnologies that are not yet widely available: babies made from the genetic material of three persons, head transplants, human cloning, and changing a baby's genetic characteristics to reduce the risk of serious diseases.

We labeled the third factor Agricultural; it comprises biotechnologies that exert power over the environment: animal testing, genetically modified foods, and pesticides.

Finally, we labeled the fourth factor Termination; it comprises biotechnologies the ethics of which turn on the consent of a relevant party to a procedure that may lead to termination of potential life: abortion and physician-assisted suicide.

\section{Manipulation Check}

Next, we examined how the disgust and fear conditions increased negative affect relative to the control condition. We averaged pleasantness ratings of the twenty IAPS pictures and regressed them on the condition factor; the neutral condition served as the reference group. The model explained substantial variance, $F(2,597)=497.2, p<.001$, Adjusted $R_{2}=.62$. The disgust pictures were rated as very unpleasant $(M=1.94, S D=0.86)$, and were significantly less pleasant than the neutral pictures $(M=4.38, S D=0.54), \mathrm{b}=-2.43$, se $=.08, t(598)=31.13, p<.001,95 \%$ CI $[-2.58,-2.27], d=-3.15$, which participants on average found mildly pleasant. The fear pictures were also more unpleasant $(M=2.76, S D=$ $.86)$ than the neutral pictures, $\mathrm{b}=-1.59, \mathrm{se}=.08, t(598)=-20.52, p<.001,95 \% \mathrm{CI}[-1.74,-$ $1.43], d=-2.06$, though less so than the disgust pictures.

\section{Induced Disgust}


We examined how fearful and disgusting photos affected biotechnology attitudes relative to observing neutral photos using a structural equation model in which two dummycoded (disgust $=1$, fear $=0$, neutral $=0$; disgust $=0$, fear $=1$, neutral $=0$ ) variables predicted each of the four biotechnology factors (see Table 2 for full unstandardized results).

Observing disgusting pictures caused a small, significant reduction in the acceptability of Existing biotechnologies relative to the neutral condition $(d=-0.23, p=.04)$. Disgust did not have a significant effect on judgments about Future biotechnologies $(d=-$ $0.01, p=.91)$, Agricultural $(d=0.10, p=.43)$, or Termination biotechnologies $(d=-0.01, p=$ .87). Adding whether participants reported being bothered by exposure to the disgust condition as a predictor of Existing biotechnologies did not change the size or significance of the effect of disgust.

Observing fearful pictures caused a nonsignificant reduction in the acceptability of Existing biotechnologies relative to the neutral condition $(d=-0.04, p=.75)$. Attitudes toward Future biotechnologies $d=0.07, p=.19)$, Agricultural $(d=0.15, p=.22)$, and Termination $(d=0.10, p=.07)$ biotechnologies were non-significantly higher in the fear condition.

The probability of committing at least one type one error when conducting eight significance tests is .34. Thus, the significant effect of disgust on attitudes toward Existing biotechnologies would not have survived a correction for multiple comparisons (e.g., a DunnSidak adjustment would have set alpha to .006).

\section{Trait Disgust}

Next, we estimated the associations between trait disgust and moral judgments of biotechnologies (see full model results in Table 3). After establishing measurement invariance of pathogen disgust across experimental conditions (see the supplemental materials), we fit a structural equation model in which the four biotechnology factors were 
regressed on pathogen disgust. Pathogen disgust had a significant negative relationship with the acceptability of Future, Agricultural, and Termination biotechnologies, but not Existing biotechnologies.

After establishing measurement invariance of sexual disgust across experimental conditions (see the supplemental materials), we ran the same model but substituted pathogen disgust with sexual disgust. Sexual disgust had a significant negative relationship with the acceptability of Existing, Future, and Termination biotechnologies, but not Agricultural biotechnologies.

\section{Interaction Between Induced and Trait Disgust}

Some researchers emphasize that not all individuals are equally affected by disgust inductions (Schnall, 2017). For instance, disgust inductions may have a greater effect on the judgments of those higher in disgust sensitivity, i.e. trait disgust (Ong, Mullette-Gillman, Kwok, and Lim, 2014). To test whether our null experimental results hold across all levels of disgust sensitivity, we conducted a set of unplanned analyses in which we tested for an interaction between trait disgust and the condition assignment. The simplest way to test an interaction between a latent variable and dummy variable is to create a (metric invariant) multi-group CFA with the dummy variable as the group factor and observe whether the strength of the association between the latent variable and outcome variable differs across levels of the group factor. We created metric invariant multi-group (with condition assignment as the group factor) versions of the models in which the biotechnology factors were regressed on pathogen disgust $(\chi 2(894)=1319.01, p<.001 ;$ RMSEA $=.049,90 \% \mathrm{CI}$ $[.043, .054] ;$ SRMR $=.066 ;$ TLI $=.895)$ and sexual disgust $(\chi 2(894)=1301.77, p<.001 ;$ RMSEA $=.048,90 \%$ CI $[.042, .053]$; SRMR $=.063$; TLI $=.903)$, respectively. For pathogen disgust, constraining the regression coefficients equal across groups did not significantly reduce fit for the model in which pathogen disgust was the exogeneous variable, $X_{2}(8)=5.98$, 
$p=.650$. Similarly, constraining the regression coefficients equal across groups did not significantly reduce model fit when sexual disgust was the exogeneous variable, $X_{2}(8)=3.17$, $p=.924$, implying no significant interactions between induced disgust and trait disgust. These findings suggest that the small and largely null results of the disgust induction are not qualified by trait disgust.

\section{Discussion}

We investigated whether disgust reduces the acceptability of biotechnologies relative to experiencing fear or no particular emotion. The results suggest that induced disgust (but not fear) had a small negative causal effect on acceptance of 'existing' biotechnologies, but not on other types of biotechnology, and that this observed small negative effect disappears after (an unplanned) correction for multiple comparisons. We are unaware of a theory which predicts that disgust will change attitudes towards Existing biotechnologies, but not other biotechnologies.

Trait disgust had more significant associations with the acceptability of biotechnologies. Pathogen disgust was negatively correlated with the acceptability of Future, Agricultural, and Termination biotechnologies, but not Existing biotechnologies. Sexual disgust was negatively associated with Existing, Future, and Termination biotechnologies, but not Agricultural biotechnologies.

\section{Implications}

Induced disgust might amplify preexisting attitudes towards Existing biotechnologies, but does not appear powerful enough to change minds. Observing disgusting photos reduced the acceptability of Existing biotechnologies by only 0.13 rating scale points (on a 1-5 scale), which is insufficient to shift responses from the "acceptable" side of the rating scale to the "unacceptable" side (May, 2014). Indeed, no item had a mean that was lower than 3 in the disgust condition or higher than 3 in the neutral condition (see Table 1). Moreover, there was 
a .34 probability we would observe at least one significant effect of the condition assignment even if neither fear nor disgust have any effects on biotechnology attitudes.

Many commentators took early research on incidental manipulations of disgust to show that emotions cause or even constitute all moral judgments (Nichols, 2004; Prinz, 2007). Our results add to the more recent consensus that induced disgust, at most, amplifies condemnation of some controversial issues. We counsel caution in drawing any positive conclusions from our data until the effect of induced disgust on Existing biotechnologies is replicated in new samples.

\section{Limitations}

One could point to the more reliable associations between trait disgust and condemnation of biotechnologies as evidence that the true effect of disgust is underestimated by the manipulation of disgust. On one version of this view, visual primes do not evoke enough disgust to affect moral judgments. Yet, recent large experiments using stronger primes such as gustatory and olfactory stimuli have also yielded null effects (Ghelfi et al., 2018; Johnson et al., 2016). Alternatively, disgust might only increase condemnation when it is evoked by the act under consideration (Wisneski \& Skitka, 2017). We are open to this possibility, but note that rejecting the relevance of incidental disgust manipulations requires discarding most experimental evidence that disgust influences moral judgments.

Common method variance could also explain why questionnaire measures of trait disgust may have stronger associations with self-reports of moral judgments than disgust inductions. Landy and Piazza (2019) found that trait disgust predicts self-reported attitudes not only toward moral violations but also imprudent behavior and even art. Controlling for response styles or supplementing self-reports with physiological markers of trait disgust would enable unbiased estimates of its relationship with biotechnology attitudes.

The biotechnology topics we used were based on previous studies. Their breadth is 
consistent with recommendations for early stages of scale development (Clark \& Watson, 2019). Our structural equation models failed tests of exact fit, though, and misspecification can bias regression coefficients. The sensitivity tests we report in the supplemental materials did not uncover any additional significant effects, which provides some assurance that underfitting the data is not responsible for our negative findings. Nevertheless, it remains possible that a more valid model of biotechnology attitudes that we did not consider would have uncovered stronger effects of disgust.

\section{Conclusion}

Our results reinforce growing doubts that the emotion of disgust is fundamental to moral judgments. Yet it could be that emotion-driven amplifications of preexisting attitudes have practical effects on policy or health outcomes. Studying how disgust affects decisions of actual patients and consumers would allow healthcare providers and advocates to assess how to best communicate the benefits and risks of biotechnologies. Meanwhile, clinicians can rest easy that graphic posters in their waiting rooms are not giving patients cold feet about procedures that may be in their best interests. 


\section{References}

Clark, L. A., \& Watson, D. (2019). Constructing validity: New developments in creating objective measuring instruments. Psychological Assessment, 31(12), 1412-1427.

Clifford, S., \& Wendell, D. G. (2016). How disgust influences health purity attitudes. Political Behavior, 38(1), 155-178.

Curry, O. S., Jones Chesters, M., \& Van Lissa, C. J. (2019). Mapping morality with a compass: Testing the theory of 'morality-as-cooperation' with a new questionnaire. Journal of Research in Personality, 78, 106-124.

De Witt, A., Osseweijer, P., \& Pierce, R. (2017). Understanding public perceptions of biotechnology through the "Integrative Worldview Framework". Public Understanding of Science, 26(1), 70-88.

Ejelöv, E., \& Luke, T. J. (2020). "Rarely safe to assume": Evaluating the use and interpretation of manipulation checks in experimental social psychology. Journal of Experimental Social Psychology, 87, 103937.

Faul, F., Erdfelder, E., Buchner, A., \& Lang, A.-G. (2009). Statistical power analyses using $\mathrm{G}^{*}$ Power 3.1: Tests for correlation and regression analyses. Behavior Research Methods, 41(4), 1149-1160.

Ghelfi, E., Christopherson, C. D., Urry, H. L., Lenne, R. L., Legate, N., Fischer, M. A., ... \& de Haan, B. (in press). Reexamining the effect of gustatory disgust on moral judgment: A multi-lab direct replication of Eskine, Kacinik, and Prinz (2011). Advances in Methods and Practices in Psychological Science.

Graham, J., Nosek, B. A., Haidt, J., Iyer, R., Koleva, S., \& Ditto, P. H. (2011). Mapping the moral domain. Journal of Personality and Social Psychology, 101(2), 366-385.

Gregorich, S. E. (2006). Do self-report instruments allow meaningful comparisons across 
diverse population groups? Testing measurement invariance using the confirmatory factor analysis framework. Medical Care, 44(11 Suppl 3), S78.

Haidt, J. (2007). The new synthesis in moral psychology. Science, 316, 998-1002.

Haidt, J., \& Joseph, C. (2004). Intuitive ethics: How innately prepared intuitions generate culturally variable virtues. Daedalus, 133(4), 55-66.

Hill, T. E. (2010). How clinicians make (or avoid) moral judgments of patients: implications of the evidence for relationships and research. Philosophy, Ethics, and Humanities in Medicine, 5(1), 11. doi:10.1186/1747-5341-5-11

Horberg, E. J., Oveis, C., Keltner, D., \& Cohen, A. B. (2009). Disgust and the moralization of purity. Journal of Personality and Social Psychology, 97(6), 963-976.

Hu, L.-T., \& Bentler, P. M. (1999). Cutoff criteria for fit indexes in covariance structure analysis: Conventional criteria versus new alternatives. Structural Equation Modeling, 6, 1-55.

Huebner, B., Dwyer, S., \& Hauser, M. (2009). The role of emotion in moral psychology. Trends in Cognitive Sciences, 13(1), 1-6.

Inbar, Y., Pizarro, D. A., \& Bloom, P. (2009). Conservatives are more easily disgusted than liberals. Cognition and Emotion, 23(4), 714-725.

Johnson, D. J., Wortman, J., Cheung, F., Hein, M., Lucas, R. E., Donnellan, M. B., ... \& Narr, R. K. (2016). The effects of disgust on moral judgments: Testing moderators. Social Psychological and Personality Science, 7(7), 640-647.

Landy, J. F., \& Goodwin, G. P. (2015). Does incidental disgust amplify moral judgment? A meta-analytic review of experimental evidence. Perspectives on Psychological Science, 10(4), 518-536.

Landy, J. F., \& Piazza, J. (2019). Reevaluating moral disgust: sensitivity to many affective 
states predicts extremity in many evaluative judgments. Social Psychological and Personality Science, 10(2), 211-219.

Libkuman, T. M., Otani, H., Kern, R., Viger, S. G., \& Novak, N. (2007). Multidimensional normative ratings for the international affective picture system. Behavior Research Methods, 39(2), 326-334.

May, J. (2014). Does disgust influence moral judgment? Australasian Journal of Philosophy, 92(1), 125-141.

Mikels, J. A., Fredrickson, B. L., Larkin, G. R., Lindberg, C. M., Maglio, S. J., \& ReuterLorenz, P. A. (2005). Emotional category data on images from the International Affective Picture System. Behavior Research Methods, 37(4), 626-630.

National Science Foundation (2012). Survey of public attitudes toward and understanding of science and technology. Retrieved from http://www.nsf.gov/statistics/srvyattitude.

Nichols, S. (2002). Norms with feeling: Towards a psychological account of moral judgment. Cognition, 84(2), 221-236.

Olatunji, B. O., Puncochar, B. D., \& Cox, R. (2016). Effects of experienced disgust on morally-relevant judgments. PloS one, 11(8), e0160357.

Ong, H. H., Mullette-Gillman, O. A., Kwok, K., \& Lim, J. (2014). Moral judgment modulation by disgust is bi-directionally moderated by individual sensitivity. Frontiers in Psychology, 5, 194. doi:10.3389/fpsyg.2014.00194

Pew Research Center. (2015). 2014 General public science survey. Retrieved from http://www.pewresearch.org.

Prinz, J. (2007). The emotional construction of morals. Oxford University Press.

R Core Team (2019). R: A language and environment for statistical computing. R Foundation for Statistical Computing, Vienna, Austria. URL https://www.R-project.org/.

Revelle, W. (2018). psych: Procedures for personality and psychological research. 
Northwestern University, Evanston, Illinois, USA, https://CRAN.R-

project.org/package $=$ psych Version $=1.8 .12$.

Rhemtulla, M., Brosseau-Liard, P. É., \& Savalei, V. (2012). When can categorical variables be treated as continuous? A comparison of robust continuous and categorical SEM estimation methods under suboptimal conditions. Psychological Methods, 17(3), 354.

Rosseel, Y. (2012). Lavaan: An R package for structural equation modeling and more. Version 0.5-12 (BETA). Journal of Statistical Software, 48(2), 1-36.

Royzman, E., Cusimano, C., \& Leeman, R. F. (2017). What lies beneath? Fear vs. disgust as affective predictors of absolutist opposition to genetically modified food and other new technologies. Judgment and Decision Making, 12(5), 466.

Schaller, M., \& Park, J. H. (2011). The Behavioral Immune System (and why it matters). Current Directions in Psychological Science, 20(2), 99-103.

Schnall, S. (2017). Disgust as embodied loss aversion. European Review of Social Psychology, 28(1), 50-94.

Schnall, S., Benton, J., \& Harvey, S. (2008). With a clean conscience: Cleanliness reduces the severity of moral judgments. Psychological Science, 19(12), 1219-1222.

Scott, S. E., Inbar, Y., \& Rozin, P. (2016). Evidence for absolute moral opposition to genetically modified food in the United States. Perspectives on Psychological Science, 11(3), 315-324.

Tracy, J. L., Steckler, C. M., \& Heltzel, G. (2019). The physiological basis of psychological disgust and moral judgments. Journal of Personality and Social Psychology, 116(1), $15-32$.

Tybur, J. M., Lieberman, D., \& Griskevicius, V. (2009). Microbes, mating, and morality: Individual differences in three functional domains of disgust. Journal of Personality and Social Psychology, 97(1), 103-122. 
van Dijke, M., van Houwelingen, G., De Cremer, D., \& De Schutter, L. (2018). So gross and yet so far away: psychological distance moderates the effect of disgust on moral judgment. Social Psychological and Personality Science, 9(6), 689-701.

Wagemans, F., Brandt, M. J., \& Zeelenberg, M. (2018). Disgust sensitivity is primarily associated with purity-based moral judgments. Emotion, 18(2), 277-289.

Wheatley, T., \& Haidt, J. (2005). Hypnotic disgust makes moral judgments more severe. Psychological Science, 16(10), 780-784.

Wisneski, D. C., \& Skitka, L. J. (2017). Moralization through moral shock: Exploring emotional antecedents to moral conviction. Personality and Social Psychology Bulletin, 43(2), 139-150. 
Table 1.

Means and Standard Deviations for Biotechnology Attitude Ratings in Each Condition

\begin{tabular}{|c|c|c|c|c|c|c|}
\hline \multirow[b]{2}{*}{ Biotechnology } & \multicolumn{2}{|c|}{$\begin{array}{l}\text { Disgust Prime } \\
\quad N=202\end{array}$} & \multicolumn{2}{|c|}{$\begin{array}{l}\text { Fear Prime } \\
N=208\end{array}$} & \multicolumn{2}{|c|}{$\begin{array}{c}\text { Neutral Prime } \\
\quad N=190\end{array}$} \\
\hline & $M$ & $S D$ & $M$ & $S D$ & $M$ & $S D$ \\
\hline Vaccine & 4.40 & 0.96 & 4.46 & 0.95 & 4.49 & 0.88 \\
\hline Eating Meat & 4.31 & 0.84 & 4.27 & 0.90 & 4.28 & 0.79 \\
\hline Alternative Medicine & 3.98 & 1.04 & 4.12 & 0.88 & 4.14 & 0.91 \\
\hline Acupuncture & 4.23 & 0.91 & 4.29 & 0.98 & 4.38 & 0.76 \\
\hline Stem Cell Therapy & 3.89 & 1.03 & 4.02 & 0.91 & 4.03 & 0.98 \\
\hline Robots in Surgery & 3.44 & 1.35 & 3.41 & 1.30 & 3.35 & 1.22 \\
\hline Testing Risky Drugs & 3.26 & 1.31 & 3.49 & 1.24 & 3.40 & 1.26 \\
\hline $\begin{array}{l}\text { GMOs to Replace } \\
\text { Gasoline }\end{array}$ & 3.45 & 1.18 & 3.76 & 1.03 & 3.72 & 1.10 \\
\hline Artificial Organs & 3.30 & 1.16 & 3.70 & 1.15 & 3.54 & 1.12 \\
\hline Animal Organ Transplant & 2.77 & 1.28 & 2.93 & 1.29 & 2.82 & 1.82 \\
\hline Animal Testing & 2.70 & 1.25 & 2.78 & 1.27 & 2.67 & 1.18 \\
\hline $\begin{array}{c}\text { Gene Edits to Reduce } \\
\text { Disease }\end{array}$ & 2.72 & 1.16 & 2.88 & 1.17 & 2.78 & 1.19 \\
\hline Three-Parent Baby & 2.20 & 1.22 & 2.32 & 1.14 & 2.17 & 1.16 \\
\hline Head Transplant & 2.00 & 1.17 & 1.95 & 1.16 & 1.97 & 1.21 \\
\hline Cloning Humans & 1.80 & 1.00 & 1.95 & 1.14 & 1.80 & 1.03 \\
\hline GMO to Improve Food & 2.73 & 1.25 & 2.77 & 1.24 & 2.76 & 1.18 \\
\hline Pesticides & 2.40 & 1.16 & 2.44 & 1.17 & 2.25 & 1.14 \\
\hline $\begin{array}{l}\text { Physician-Assisted } \\
\text { Suicide }\end{array}$ & 2.86 & 1.39 & 3.17 & 1.41 & 2.91 & 1.21 \\
\hline Abortion & 2.92 & 1.39 & 3.04 & 1.45 & 2.86 & 1.42 \\
\hline Vaccine & 4.40 & 0.96 & 4.46 & 0.95 & 4.49 & 0.88 \\
\hline Obese Paying More & 1.90 & 1.08 & 2.10 & 1.21 & 1.92 & 1.06 \\
\hline
\end{tabular}

Notes: Participants 5-point rating scale $(1=$ Completely acceptable; $2=$ Unacceptable; $3=$ Neither acceptable nor unacceptable; $4=$ Acceptable; $5=$ Completely acceptable). We defined "moral acceptability" for participants as "something that, from your moral viewpoint, a person can do without being wrong." 
Table 2.

Latent mean differences between emotion and neutral conditions

$\begin{array}{llllll}b & \text { se } & Z & p & 95 \% \mathrm{CI} & d\end{array}$

Disgust vs. Neutral

\begin{tabular}{lcccccc} 
Existing & -0.13 & 0.06 & -2.01 & .044 & {$[-0.25,-0.00]$} & -0.23 \\
Future & -0.01 & 0.10 & -0.12 & .908 & {$[-0.21,0.18]$} & -0.01 \\
Agricultural & 0.09 & 0.11 & 0.80 & .426 & {$[-0.13,0.30]$} & 0.10 \\
Termination & -0.02 & 0.13 & -0.17 & .867 & {$[-0.28,0.24]$} & -0.01 \\
\hline
\end{tabular}

Fear vs. Neutral

$\begin{array}{lllllll}\text { Existing } & -0.02 & 0.06 & -0.32 & .750 & {[-0.13, .010]} & -0.04\end{array}$

$\begin{array}{lllllll}\text { Future } & 0.13 & 0.10 & 1.31 & .191 & {[-0.07,0.33]} & 0.07\end{array}$

$\begin{array}{lllllll}\text { Agricultural } & 0.13 & 0.10 & 1.22 & .221 & {[-0.08,0.33]} & 0.15\end{array}$

$\begin{array}{lllllll}\text { Termination } & 0.24 & 0.13 & 1.83 & .067 & {[-0.02,0.50]} & 0.10\end{array}$

Notes: Model fit: $\chi 2(161)=288.47, p<.001$; RMSEA $=.036,90 \%$ CI [.030, .043]; SRMR $=$ $.036 ; \mathrm{TLI}=.941$. Results are from a structural equation model in which two dummy-coded (disgust $=1$, fear $=0$, neutral $=0$; disgust $=0$, fear $=1$, neutral $=0$ ) variables predicted each of the four biotechnology factors. 
Table 3.

Latent effect of trait disgust on biotechnology attitudes

\begin{tabular}{lcccccc}
\hline & $b$ & $s e$ & $Z$ & $p$ & $95 \% \mathrm{CI}$ & $r$ \\
\hline Pathogen Disgust & & & & & & \\
Existing & -0.03 & 0.03 & -1.12 & .262 & {$[-0.09,0.02]$} & -.07 \\
Future & -0.10 & 0.05 & -2.17 & .030 & {$[-0.19,-0.01]$} & -.12 \\
Agricultural & -0.13 & 0.04 & -2.89 & .004 & {$[-0.21,-0.04]$} & -.17 \\
Termination & -0.13 & 0.06 & -2.21 & .027 & {$[-0.25,-0.02]$} & -.13 \\
Model fit: $\chi 2(274)=524.36, p$ & $<.001 ;$ RMSEA $=.039,90 \%$ CI $[.033, .044] ;$ \\
SRMR = $043 ;$ TLI $=.928$ & & & & &
\end{tabular}

\section{Sexual Disgust}

$\begin{array}{lcccccc}\text { Existing } & -0.04 & 0.02 & -2.07 & .038 & {[-0.08,-0.00]} & -.11 \\ \text { Future } & -0.17 & 0.04 & -5.02 & <.001 & {[-0.24,-0.11]} & -.28 \\ \text { Agricultural } & -0.04 & 0.03 & -1.31 & .189 & {[-0.10,0.02]} & -.07 \\ \text { Termination } & -0.28 & 0.04 & -6.40 & <.001 & {[-0.37,-0.19]} & -.36\end{array}$

Model fit: $\chi 2(274)=574.45, p<.001 ;$ RMSEA $=.043,90 \%$ CI [.038, .047];

SRMR $=.042 ; \mathrm{TLI}=.919$

Notes: Results are from structural equation models in which the biotechnology factors, pathogen disgust, and sexual disgust were all treated as latent variables. In the first model, the four biotechnology factors were regressed on pathogen disgust; in the second model, the biotechnology factors were regressed on sexual disgust. 